\title{
Preliminary results of the heavy-light meson spectrum using chirally improved light quarks
}

\author{
T. Burch ${ }^{a *}$, C. Gattringer a and A. Schäfer ${ }^{\mathrm{a}}$ \\ anstitut für Theoretische Physik, Universität Regensburg, D-93040 Regensburg, Germany
}

Using a "wall" of quark point sources, we invert the chirally improved Dirac operator to create an "incoherent" collection of quark propagators which originate from all spatial points of the source time slice. The lowest-order NRQCD approximation is used to create heavy-quark propagators from the same wall source. However, since the numerical cost involved in computing such heavy-quark propagators is low, we are able to use a number of source gauge paths to establish coherence between the heavy and light quarks at several spatial separations. The resulting collection of heavy-light meson correlators is analyzed to extract the corresponding mass spectrum.

\section{Light-quark source and propagators}

For the light quarks, we use the chirally improved Dirac operator, constructed via an expansion in gauge paths and the corresponding approximate solution of the Ginsparg-Wilson relation (for details see Ref. [1]).

As sources for the light-quark propagators, we use 12 (3 colors $\times 4$ spins $)$ uniform walls, setting the color and spin of the quarks to be the same at all spatial positions and relying upon the random gauges of the ensemble of configurations to cancel any non-gauge-invariant (open) contributions to hadron correlators constructed from these quark propagators. This is similar to the volume source used by others [2] for disconnected correlators, except for the fact that our source sits on only a single time-slice. Connected meson correlators from two of these quarks should exhibit a signal arising from $L^{3}$ closed (or gauge-invariant) correlators, along with some additional noise from the $L^{3}\left(L^{3}-1\right)$ open correlators. These sources have been used before for heavy quarkonium correlators with some success [3] since the signal-tonoise ratio proved to be surprisingly good. Since we retain our light-quark propagators and the heavy-quark evolution is computationally cheap, we are free to use a number of different gauge paths to create "walls" of extended heavy-light

${ }^{*}$ Presented by T. Burch. meson sources from the light-quark source before we compute the heavy-quark propagators. In this way we can construct a full matrix of correlators with various spatial extents and a collection of starting positions without additional light-quark inversions.

\section{Heavy-quark evolution}

The heavy-quark propagators are created using lowest-order NRQCD, along with some improvements for the lattice version [4. A single timestep of heavy-quark evolution is achieved via

$$
\begin{aligned}
G_{Q}(\vec{x}, 0 \mid \vec{y}, t+a) & =\left(1-\frac{a \tilde{H}_{0}}{2 n}\right)_{t+a}^{n} U_{4}^{\dagger}\left(\vec{y}^{\prime \prime}, t\right) \\
& \left(1-\frac{a \tilde{H}_{0}}{2 n}\right)_{t}^{n} G_{Q}\left(\vec{x}, 0 \mid \vec{y}^{\prime}, t\right),
\end{aligned}
$$

where we use $n=2$ (this is sufficient for $b$ quarks on our lattices) and the Hamiltonian is the $O\left(a^{2}\right)$ improved, covariant, kinetic-energy operator, corrected to remove $O(a)$ artifacts in the time evolution:

$\tilde{H}_{0}=-\frac{\tilde{\Delta}^{2}}{2 m_{Q}}-\frac{a \tilde{\Delta}^{4}}{16 n m_{Q}^{2}}$.

Since we include only the kinetic-energy term in our NRQCD expansion, there is no coupling of 
the heavy quark's color and spin, the latter being provided simply by the projection

$G_{\alpha \beta ; Q}^{a b}(\vec{x}, 0 \mid \vec{y}, t)=\frac{1}{2}\left(1+\gamma_{4}\right)_{\alpha \beta} G_{Q}^{a b}(\vec{x}, 0 \mid \vec{y}, t)$.

\section{Source and sink operators}

The gauge paths and corresponding spin structures we use to create our mesons can be found in Refs. [56]. Through sums $(s)$ and differences $(p)$ of paths in opposite directions, along with different Dirac matrices, meson operators with different symmetries can be constructed: e.g.,

$$
\begin{aligned}
& S: \bar{Q}(x) \Gamma U_{P}\left(x \mid x^{\prime}\right) q\left(x^{\prime}\right)=\bar{Q} \gamma_{5} \sum_{i} s_{i} q \\
& P_{-}: \bar{Q} \sum_{i} \gamma_{i} p_{i} q ; P_{+}: \bar{Q}\left(\gamma_{1} p_{1}-\gamma_{2} p_{2}\right) q ; \\
& D_{ \pm}: \bar{Q} \gamma_{5}\left(s_{1}-s_{2}\right) q .
\end{aligned}
$$

Thus far, we have only included straight paths, limiting us to S-, P- and D-wave mesons. Also, since we don't include heavy-quark spin interactions, a number of physical states are not separable: the S-wave operator, $S$, must be viewed as creating a spin-average of $J^{P}=0^{-}$and $1^{-}$states; the $P_{-}, J^{P}=\left\{0^{+}, 1^{+}\right\}$; the $P_{+}, J^{P}=\left\{1^{+}, 2^{+}\right\}$; and the $D_{ \pm}, J^{P}=\left\{1^{-}, 2^{-}, 3^{-}\right\}$.

\section{Heavy-light correlator matrix}

Given all the ingredients, we now present the form of our heavy-light correlators:

$$
\begin{aligned}
C_{i j}(t)= & \left\langle\sum_{\vec{x}, \vec{x}^{\prime}, \vec{y}}\left[\Gamma_{\delta \alpha} U_{P}^{d a}\left(x^{\prime} \mid x^{\prime \prime}\right)\right]_{i} G_{\alpha \beta ; q}^{a b}(\vec{x}, 0 \mid \vec{y}, t)\right. \\
& {\left.\left[\Gamma_{\beta \gamma}^{\prime *} U_{P^{\prime}}^{* b c}\left(y \mid y^{\prime}\right)\right]_{j} G_{\gamma \delta ; \bar{Q}^{c}}^{c d}\left(\vec{y}^{\prime}, t \mid \vec{x}^{\prime}, 0\right)\right\rangle \cdot(7) }
\end{aligned}
$$

Note the sum over both quark source positions, $\vec{x}$ and $\vec{x}^{\prime}$, due to the use of the walls; only the closed terms (where $\vec{x}=\vec{x}^{\prime \prime}$ ) will contribute to the signal. We include both the $\bar{Q} q$ and $Q \bar{q}$ correlators from opposite time directions.

After calculating the matrix of correlators, $C_{i j}(t)$, between our linearly independent meson operators, $\psi_{i}=\bar{Q}(x) \Gamma U_{P}\left(x \mid x+r_{i}\right) q\left(x+r_{i}\right)$, we solve the corresponding generalized eigenvalue problem to extract the physical states [7]:

$\sum_{j} C_{i j}(t) \psi_{j}^{k}=\lambda^{k}\left(t, t_{0}\right) \sum_{j} C_{i j}\left(t_{0}\right) \psi_{j}^{k}$,

where, for large enough values of $t\left(\gg t_{0}\right)$,

$\lambda^{k}\left(t, t_{0}\right)=e^{-m_{k}\left(t-t_{0}\right)}$.

So the eigenvalues provide the masses of the states and the eigenvectors reveal the meson wavefunctions' dependencies upon the $\bar{Q} q$ separation, $r_{i}(=0-7 a$ along the principal axes). This technique of using operators with different spatial extents has also been recently used to determine excited states of baryons [8].

\section{Preliminary results}

In this section we present results from one set (100) of $12^{3} \times 24$ quenched configurations which were created using the Lüscher-Weisz action at $\beta=7.9\left(r_{0} / a=3.384 \rightarrow a^{-1} \approx 1330 \mathrm{MeV}\right)$.

In Fig. 1 we plot the effective masses calculated from the largest eigenvalues of each set of operators. The horizontal lines indicate the $1 \sigma$ masses resulting from correlated fits to the data over the corresponding time ranges. We repeat this for 6 mass combinations $\left(a m_{Q}=3.0,3.5\right.$ and $\left.a m_{q}=0.04,0.08,0.10\right)$ in order to interpolate to the physical values of $m_{b}$ and $m_{s}$, and to extrapolate to $m_{u(d)}$. The physical strange quark mass is determined via light-light meson spectroscopy $\left(M_{s \bar{s}}^{2} \approx 2 M_{K}^{2}-M_{\pi}^{2} \rightarrow a m_{s} \approx 0.09\right)$; the bottom quark mass is determined via finite-momentum heavy-light states and the corresponding dispersion relation:

$E(p) \approx E_{0}+\frac{\vec{p}^{2}}{2 M}$,

where we require that $M=\left(3 M_{B_{s}^{*}}+M_{B_{s}}\right) / 4$. At this lattice spacing we find $m_{b} \approx 4240 \mathrm{MeV}$. The interpolated (and extrapolated) results for the $B_{s}^{(*)}\left(B^{(*)}\right)$ system are displayed in Table 1 (Table 2).

The $S P$ and $S D$ mass splittings are in agreement with experiment (available only for the former) and the findings of previous lattice studies 61011]. All ground-state eigenvector components have the same sign: i.e., there are no 


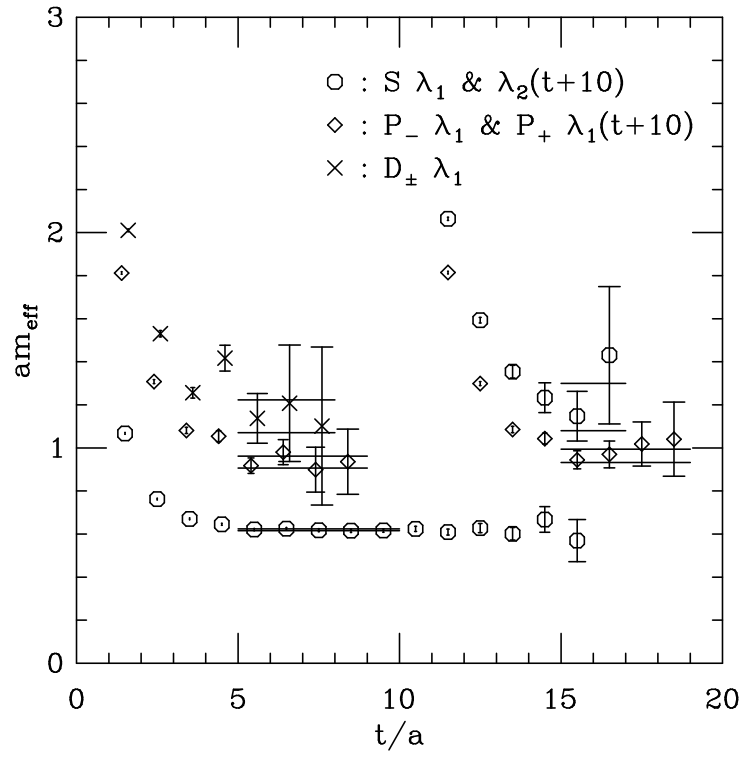

Figure 1. Effective masses versus time from the largest eigenvalues of each of the meson operators. The results for the $P_{+}$and for the first-excited state of the $S$ are shifted by 10 in time for clarity. $a m_{q}=0.08, a m_{Q}=3.0$ and $t_{0} / a=1$.

nodes in the corresponding ground-state heavylight wavefunctions. The first-excited S-wave displays a single node at $r \approx 2 a \approx 0.6 r_{0}\left(r \approx 0.5 r_{0}\right.$ found in 6]). Our mass results for the firstexcited S-wave, however, appear to be higher than those found previously ( $\approx 500 \mathrm{MeV}$ for $B_{s}$ ). Our mass plateaus for this state are rather suspect (typically only $3 t$ values were used in the fits). This issue can only be resolved with better statistics, which can be achieved a number of ways: more or larger configurations, more wall sources with locally gauge-rotated quark fields, more operators (e.g., non-straight paths). We would like to consider each of these options in the future, along with the inclusion of heavy-quark spin interactions to resolve more spin splittings in these heavy-light systems.

This work is supported by BMBF and GSI. All computations were performed on the Hitachi SR8000 in Munich and at the RZ Regensburg.
Table 1

Mass splittings for the $B_{s}^{(*)}$ system.

\begin{tabular}{llll}
\hline $\mathcal{O}$ & $a\left(M-M_{S}\right)$ & $M-M_{S}(\mathrm{MeV})$ & exp. $[9]$ \\
\hline$S^{\prime}$ & $0.58(11)$ & $770(150)(?)$ & - \\
$P_{-}$ & $0.314(28)$ & $419(37)$ & $448(16)$ \\
$P_{+}$ & $0.341(31)$ & $455(41)$ & $448(16)$ \\
$D_{ \pm}$ & $0.530(76)$ & $710(100)$ & - \\
\hline
\end{tabular}

Table 2

Mass splittings for the $B^{(*)}$ system.

\begin{tabular}{llll}
\hline $\mathcal{O}$ & $a\left(M-M_{S}\right)$ & $M-M_{S}(\mathrm{MeV})$ & exp. [9] \\
\hline$S^{\prime}$ & $0.46(17)$ & $610(230)(?)$ & - \\
$P_{-}$ & $0.306(50)$ & $408(67)$ & $384(8)$ \\
$P_{+}$ & $0.330(58)$ & $440(77)$ & $384(8)$ \\
$D_{ \pm}$ & $0.45(11)$ & $600(150)$ & - \\
\hline
\end{tabular}

\section{REFERENCES}

1. P. H. Ginsparg and K. G. Wilson, Phys. Rev. D25 (1982) 2649; C. Gattringer, Phys. Rev. D63 (2001) 114501; C. Gattringer, I. Hip and C. B. Lang, Nucl. Phys. B597 (2001) 451.

2. Y. Kuramashi et al., Phys. Rev. Lett. 72 (1994) 3448; F. Farchioni, G. Münster and R. Peetz, hep-lat/0404004

3. T. Burch, K. Orginos and D. Toussaint, Phys. Rev. D64 (2001) 074505; Nucl. Phys. Proc. Suppl. 106 (2002) 382.

4. G. P. Lepage et al., Phys. Rev. D46 (1992) 4052.

5. P. Lacock et al., Phys. Rev. D54 (1996) 6997.

6. C. Michael and J. Peisa, Phys. Rev. D58 (1998) 034506.

7. C. Michael, Nucl. Phys. B259 (1985) 58; M. Lüscher and U. Wolff, Nucl. Phys. B339 (1990) 222.

8. T. Burch et al., hep-lat/0405006 BGR Collaboration, these proceedings, hep-lat/0409014.

9. S. Eidelman et al., Phys. Lett. B592 (2004) 1.

10. A. Ali Khan et al., Phys. Rev. D62 (2000) 054505 .

11. A. M. Green et al., Phys. Rev. D69 (2004) 094505 . 